anaesthetics were admitted as day patients in all but two centres, thus avoiding unnecessary expense to the hospital and disruption to the patient. In most cases these anaesthetics were given by junior anaesthetists.

Most non-anaesthetic staff did not starve their patients before giving Bier's blocks or intravenous sedation. Cardiorespiratory arrest is a recognised problem with both these techniques, and the outcome is adversely affected by aspiration; thus starvation is advisable. As monitoring equipment was universally available it would seem sensible to use it. Reduction of Colles' fractures under local haematoma block avoids many of the problems associated with anaesthesia, but the price is increased pain to the patient during reduction, which may not be acceptable. ${ }^{3}$
Despite our findings there were few important complications reported in the year preceding our study, and no hospitals had changed their method of anaesthesia in the light of the confidential enquiry into perioperative deaths. We therefore conclude that all of the above methods are safe and efficient but urge staff in accident departments to be vigilant over simple precautions to keep them so.

1 Ralis ZA. Epidemics of fractures during periods of snow and ice. Br Med $\mathcal{J}$
$1986 ; 293: 484$.

2 Buck N, Devlin HB, Lunn JN. Report of a confidential enquiry into perioperative deaths. London: Nuffield Hospitals Trust, 1987.

3 Cobb AG, Houghton GR. Local anaesthetic infiltration versus Bier's block for Colles' fractures. Br Med f 1985;291:1683-4.

(Accepted 12 September 1989)

\title{
Spontaneous abortion rates after natural and assisted conception
}

\author{
Christopher Steer, Stuart Campbell, \\ Melanie Davies, Bridgett Mason, \\ William Collins
}

\section{Bourne-Hallam Medical Centre, London W1N 5LR Christopher Steer, MRCOG, research fellow \\ Melanie Davies, MRCOG, research fellow \\ Bridgett Mason, MRCS, medical director}

Department of Obstetrics and Gynaecology, King's College School of Medicine and Dentistry, London SE5 8RX

Stuart Campbell, FRCOG, professor of obstetrics and gynaecology

William Collins, DSC, professor of reproductive biochemistry

Correspondence to: $\mathrm{Dr}$ Steer.

BrMed f 1989;299:1317-8
Many studies have determined the incidence of spontaneous abortion in women, and it has been suggested that the rate after assisted conception is higher than that during natural pregnancy. ${ }^{1}$ Problems in interpreting results can, however, arise from the use of different reference points to estimate gestational age and from the availability of alternative and complementary methods for diagnosing an early pregnancy. We therefore determined the rate of spontaneous abortion among 430 women undergoing assisted conception and compared it with data from other series.

\section{Methods and results}

The 430 women became pregnant after in vitro fertilisation and embryo transfer at the Hallam Medical Centre from July 1984 to August 1987. The day of oocyte retrieval (follicular rupture) was used as the reference point for calculating gestational age at the time each test was applied. A serum concentration of human chorionic gonadotrophin of over $25 \mathrm{U} / \mathrm{l}$, as determined by radioimmunoassay, was used as the index of early pregnancy 16 days after follicular rupture. Transabdominal ultrasonography was used to identify a gestational sac at 28 days' (4 weeks') gestation from follicular rupture and a fetal heart at 35 days' ( 5 weeks') gestation.

Twenty six $(6 \%)$ pregnancies were shown to be ectopic by 28 days' gestation and were terminated. The remainder (404) were all diagnosed initially from raised serum human chorionic gonadotrophin concentrations. Overall $93(23 \%)$ of these pregnancies ended in spontaneous abortion. Thirty four women were lost to the study at 28 days, but 370 underwent ultrasonography, which showed the presence of a gestational sac. Forty nine $(13 \%)$ of these pregnancies ended in spontaneous abortion. Another 40 women were lost at 35 days, but 330 underwent a second ultrasonic scan, which showed the presence of a fetal heart. Twelve (3.6\%) of these pregnancies ended in spontaneous abortion, which is similar to the rate of $3.7 \%$ reported after natural conceptions. ${ }^{2}$

We compared our data with those reported by others (17 references available from the authors) giving a total of 6710 conceptions (4604 natural, 1700 after in vitro

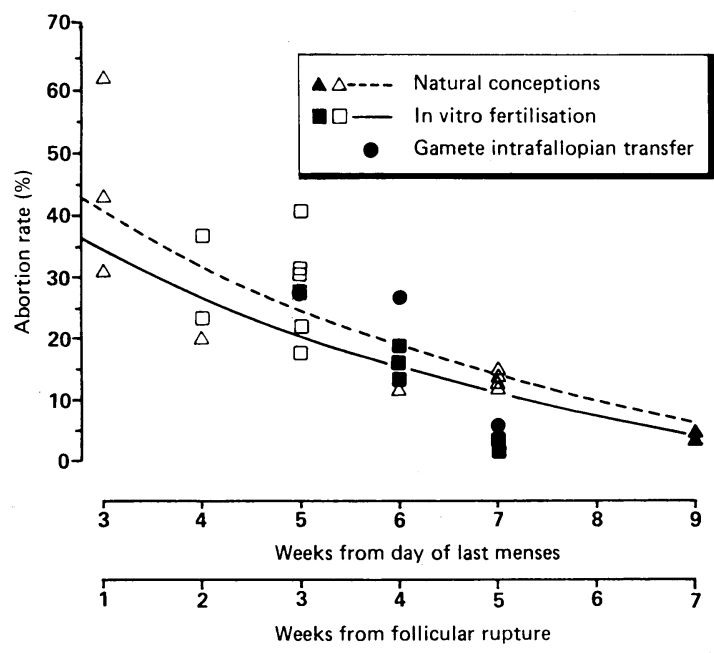

Relations between spontaneous abortion rate and gestational age at which diagnostic test was applied for natural and assisted conceptions. Closed symbols represent studies in which diagnosis was based on ultrasonography

fertilisation and embryo transfer, and 406 after gamete intrafallopian transfer). The spontaneous abortion rates for these three groups of women from each study are shown in the figure. The gestational ages were either related to the presumed time of ovulation (follicular rupture) or day 1 of the last menses. The relation between abortion rate, gestational age, and type of conception was derived from a model using analysis of covariance with weighting to allow for the different number of pregnancies in each study. An allowance was also made for the heterogeneity among studies. A linear relation was found between the abortion rate, $p$, expressed as $\log p /(1-p)$ and gestational age. The derived values for natural conceptions and those from in vitro fertilisation are shown. The effect of gestational age was highly significant $(p<0.0001)$. The test of parallelism was non-significant $(p=0 \cdot 4)$, showing that there was no difference in abortion rates between the two groups at different stages of gestation. There were no significant differences between the abortion rates for natural conceptions and those from in vitro fertilisation or for natural conceptions and those from gamete intrafallopain transfer $(p=0.20$ and 0.08 respectively). There was, however, a significant difference between in vitro fertilisation and gamete intrafallopian transfer $(p=0 \cdot 02)$, which might be explained by the inclusion of 193 women aged over 40 among the 1071 in the series reported by Craft et al ${ }^{3}$ (spontaneous abortion rate $27 \cdot 2 \%$ ). The $95 \%$ confidence intervals for abortion rates at 5 weeks' gestation from follicular rupture (or 7 weeks' from last menses) were: natural conception $7 \cdot 4 \%$ to $26 \cdot 1 \%$, in vitro fertilisation $6 \cdot 2 \%$ to $20.0 \%$, and gamete intrafallopian transfer $10 \cdot 2 \%$ to $43 \cdot 3 \%$. 


\section{Comment}

We believe that the previous report of a higher abortion rate after in vitro fertilisation might be related to the methods used for diagnosing early pregnancy. Our data suggest that the most effective way of diagnosing a viable pregnancy after assisted conception is by detecting a fetal heart at 4 to 6 weeks' gestation from follicular rupture. This method also minimises the psychological impact of pregnancy testing for the patient. Most spontaneous abortions, however, occur before this development, and biochemical indices of embryo viability are required to identify the problems and improve the efficacy of assisted conception. We conclude that the rate of spontaneous abortion following in vitro fertilisation and embryo transfer is not significantly different from that observed after natural conception.

The analysis of covariance was undertaken by J P Royston, Department of Medical Physics, Royal Postgraduate Medical School, London.

1 Liu H-C, Jones HW, Rosenwaks $Z$. The efficiency of human reproduction after in vitro fertilization and embryo transfer. Fertil Steril 1988;49:649-53.

2 Simpson JL, Mills LJL, Holmes LB, Ober CL, Aarons J. Low fetal loss rates after ultrasound-proved viability in early pregnancy. $\mathcal{F}$ AMA 1987;258:2555-

3 Craft I, Al-Shawaf T, Lewis P, et al. Analysis of 1071 GIFT procedures: the case for a flexible approach to treatment. Lancet 1988;ii:1094-8 .

(Accepted 11 September 1989)

\section{Gold treatment for kala-azar}

\section{P Singh, M Mishra, A B Khan, S L Ramdas, S Panjiyar}

\section{Darbhanga Medical \\ College and Hospital, Darbhanga, Bihar 846003, India \\ M P Singh, MD, assistant professor of medicine M Mishra, FRCP, professor and head, department of medicine}

A B Khan, MD, professor and head, department of pathology S L Ramdas, MD, resident medical officer, department of medicine

S Panjiyar, MB, assistant health officer

Correspondence and requests for reprints to: Professor Mishra.
Gold has long been used to treat patients with rheumatoid arthritis, though it is not clear exactly how it works. After administration it is deposited in organs that are rich in mononuclear phagocytes.' The amastigote form of leishmania also selects macrophages. We therefore wondered if gold could be used to treat patients with kala-azar.

\section{Patients, methods, and results}

Because of its possible toxicity we gave gold only to patients who had relapsed after treatment or had failed to respond to treatment with sodium stibogluconate for kala-azar. We treated six women and four men, mean (SD) age $23 \cdot 2(10 \cdot 8)$ years and mean (SD) weight $35 \cdot 8$ $(10 \cdot 2) \mathrm{kg}$ (table). We obtained informed consent from all 10 patients after explaining the possible toxic effects of gold. We carried out thorough clinical examinations and routine investigations before treating them with sodium aurothiomalate $10 \mathrm{mg}$ intramuscularly as the first dose followed by $20 \mathrm{mg}$ intramuscularly on alternate days to a total dose of $250 \mathrm{mg}$. The patient in case 9 , weighing $18 \mathrm{~kg}$, was given $10 \mathrm{mg}$ on alternate days to a total dose of $200 \mathrm{mg}$. A bone marrow aspirate obtained by puncture of the iliac crest and stained with Leishman's stain showed Leishman-Donovan bodies in all 10 patients. None of the patients had cardiac, pulmonary, renal, or hepatic complications.

Haematological investigations were repeated weekly and a final assessment made at the end of four weeks when the bone marrow was re-examined for the presence of leishmania. Liver enlargement was noted in the midclavicular line, and the spleen was measured along its long axis from the costal margin in the anterior axillary line to its tip.

All 10 patients responded clinically; their fevers abated in six to 13 days and the size of the liver and spleen regressed. The white cell count and haemoglobin concentration, however, showed little change, while the total platelet count actually dropped. In three patients (cases 4, 5, and 7) Leishman-Donovan bodies were seen after four weeks, but two weeks later, with no further treatment, no parasites were seen in the bone marrow of the patient in case 4 . In one patient (case 9) the bone marrow could not be re-examined at the end of four weeks, but at follow up three months later the patients in cases 5,7 , and 9 also became parasitologically negative, and the total platelet count returned to normal in all. All 10 patients remained well one year after discharge from hospital.

\section{Comment}

All 10 patients showed an excellent clinical response to treatment with sodium aurothiomalate, their temperatures all returning to normal and the size of the liver and spleen regressing. The fall in the total platelet count might be explained by an increased elimination of thrombocytes rather than overall depression of the bone marrow.

We suggest that gold treatment should be considered for patients who have so far been treated with amphotericin B or pentamidine, as both are toxic, and such patients may tolerate treatment with gold.

We thank Mr Amar Nath Jha for typing the manuscript.

1 Flower RJ, Moncada S, Vane JR. Drug therapy of inflammation. In: Gilman AG, Goodman LS, Rall TW, Murad F, eds. Goodman and Gilman's the pharmacological basis of therapeutics. New York: Macmillan, 1985:704-7.

(Accepted 17 August 1989)

Findings in 10 patients before and four weeks after starting treatment with gold

\begin{tabular}{|c|c|c|c|c|c|c|c|c|c|c|c|}
\hline Case No: & 1 & 2 & 3 & 4 & 5 & 6 & 7 & 8 & 9 & 10 & Mean \\
\hline Age (years) & 13 & 40 & 14 & 18 & 35 & 35 & 12 & 22 & 13 & 30 & $23 \cdot 2$ \\
\hline Weight $(\mathrm{kg})$ & 35 & 35 & 28 & 41 & 35 & 38 & 27 & 48 & 18 & 53 & $35 \cdot 8$ \\
\hline Previous sodium stibogluconate treatment $(\mathrm{g})$ & 30 & 18 & 30 & 12 & 36 & 18 & 27 & 18 & 15 & 18 & 17 \\
\hline \multicolumn{12}{|l|}{ Enlarged liver $(\mathrm{cm})$ : } \\
\hline Before treatment & 10 & 10 & 6 & 4 & 5 & 5 & 8 & 8 & 4 & 7 & $6 \cdot 7$ \\
\hline After treatment & 4 & 5 & 2 & 2 & 3 & 2 & 4 & 2 & 2 & 3 & $2 \cdot 9^{\star}$ \\
\hline \multicolumn{12}{|l|}{ Enlarged spleen $(\mathrm{cm})$ : } \\
\hline Before treatment & 19 & 11 & 18 & 16 & 19 & 9 & 17 & 15 & 12 & 15 & $15 \cdot 1$ \\
\hline After treatment & 13 & 3 & 9 & 8 & 8 & 2 & 11 & 4 & 9 & 10 & $7 \cdot 7 \star$ \\
\hline \multicolumn{12}{|l|}{ Total leucocyte count $\left(\times 10^{\%} / 1\right)$ : } \\
\hline Before treatment & $5 \cdot 2$ & 2 & $4 \cdot 7$ & $4 \cdot 8$ & 3.6 & $5 \cdot 1$ & 1.85 & $3 \cdot 7$ & $6 \cdot 4$ & $2 \cdot 9$ & $4 \cdot 03$ \\
\hline After treatment & $4 \cdot 2$ & $5 \cdot 2$ & $4 \cdot 0$ & $4 \cdot 5$ & $4 \cdot 2$ & $4 \cdot 3$ & $2 \cdot 75$ & $5 \cdot 2$ & $5 \cdot 1$ & $7 \cdot 4$ & $4 \cdot 7$ \\
\hline \multicolumn{12}{|l|}{ Total platelet count $\left(\times 10^{4} / 1\right)$ : } \\
\hline Before treatment & 185 & 110 & 210 & 180 & 225 & 250 & 190 & 150 & 250 & 150 & 190 \\
\hline After treatment & 168 & 80 & 175 & 170 & 180 & 180 & 145 & 70 & 180 & 110 & $145 \cdot 8^{\star}$ \\
\hline \multicolumn{12}{|l|}{ Haemoglobin $(\mathrm{g} / \mathrm{l})$ : } \\
\hline Before treatment & 102 & 110 & 94 & 92 & 52 & 94 & 60 & 100 & 96 & 90 & 89 \\
\hline After treatment & 105 & 112 & 100 & 100 & 55 & 100 & 68 & 110 & 124 & 122 & $99 \cdot 6$ \\
\hline No of days till fever abated & 6 & 8 & 11 & 10 & 10 & 10 & 13 & 12 & 13 & 8 & $10 \cdot 1$ \\
\hline Amastigotes in bone marrow after treatment & None & None & None & Yes & Yes & None & Yes & None & Not done & None & \\
\hline
\end{tabular}

*Compared to pretreatment value $\mathrm{p}<0.001$ ( $t$ test for paired data). 